\title{
Molecular mechanisms of leukotriene synthesis: the changing paradigm
}

\author{
M. PETERS-GOLDEN
}

Pulmonary and Critical Care Medicine Division, Department of Internal Medicine, University of Michigan Medical School, Ann Arbor, MI, USA

Leukotrienes (LTs) are potent bioactive lipids which have been conventionally viewed as paracrine mediators of inflammatory disease processes such as asthma [1]. More recent information suggests that they are also important participants in disease processes characterized by cellular proliferation and fibrogenesis [2-4], and that they subserve a homeostatic role in antimicrobial host defense [5]. In view of the actions and importance of LTs, substantial effort over the last several years has been directed at increasing our understanding of their synthesis. Here I will provide a brief review of these recent advances in LT synthesis, with an emphasis on the biochemistry, molecular biology, and cell biology of the key enzymes involved. Certain unexpected findings which have emerged from these studies underscore the appropriateness of a perspective on LT biology which transcends conventional notions.

\section{Overview of the leukotriene synthetic pathway}

(see Fig. 1)

Leukotriene synthesis can be triggered by a variety of soluble and particulate stimuli, including antigens, microbes, cytokines, immune complexes, and model agonists such as calcium ionophores. These result in the activation of signal transduction cascades and the generation of second messengers such as $\mathrm{Ca}^{2+}$, which in turn activate phospholipase $\mathrm{A}_{2}\left(\mathrm{PLA}_{2}\right)$. This enzyme initiates LT synthesis by catalysing the hydrolysis of arachidonic acid (AA) from membrane phospholipids. Although there are multiple isoforms of $\mathrm{PLA}_{2}$ [6], the most attractive candidate to subserve this function is cytosolic $\mathrm{PLA}_{2}$ $\left(\mathrm{cPLA}_{2}\right)$, a $\mathrm{Ca}^{2+}$-dependent and AA-preferring $85 \mathrm{kDa}$ enzyme.

The liberated free AA can then be acted on by the first committed enzyme in the LT synthetic pathway, 5lipoxygenase (5-LO). This $78 \mathrm{kDa}$ protein catalyses a twostep reaction: oxygenation of $\mathrm{AA}$ at carbon 5 to form an

Correspondence: Dr M. Peters-Golden, Pulmonary and Critical Care Medicine Division, Department of Internal Medicine, University of Michigan Medical School, 6301 MSRB III, Box 0642, Ann Arbor, MI 48109, USA.

(C) 1998 Blackwell Science Ltd unstable intermediate, 5-hydroperoxyeicosatetraenoic acid (5-HPETE), followed by dehydration of 5-HPETE to the epoxide leukotriene $\mathrm{A}_{4}\left(\mathrm{LTA}_{4}\right)$. Maximal activity of 5-LO requires $\mathrm{Ca}^{2+}$, ATP, and hydroperoxide, and its efficient utilization of endogenously released AA in intact cells requires a $18 \mathrm{kDa}$ helper protein, termed 5-LO activating protein (FLAP) [7]. FLAP is an AA-binding protein which is thought to optimally 'present' substrate to 5-LO. LTA 4 is the precursor for the stable bioactive LTs. It can be hydrolysed by $\mathrm{LTA}_{4}$ hydrolase to $\mathrm{LTB}_{4}$, which has potent chemotactic and leucocyte-activating effects, or conjugated with reduced glutathione by $\mathrm{LTC}_{4}$ synthase to yield $\mathrm{LTC}_{4}$; $\mathrm{LTC}_{4}$ can be further modified extracellularly by sequential amino acid removal to yield $\mathrm{LTD}_{4}$ and $\mathrm{LTE}_{4}$. Collectively, $\mathrm{LTC}_{4}, \mathrm{D}_{4}$, and $\mathrm{E}_{4}$ are known as the cysteinyl LTs, and comprise the smooth muscle contractile and vascular permeability activities long recognized as slow-reacting substance. Examination of this pathway thus identifies several critical proteins which are potential loci for regulation of LT synthesis, as will be discussed.

\section{Cellular sources of leukotrienes}

Phospholipase $\mathrm{A}_{2} \mathrm{~s}$, including $\mathrm{cPLA}_{2}$, are ubiquitously expressed among various cell types. However, 5-LO and FLAP proteins are largely restricted to cells of bone marrow origin (myeloid cells), and it is these cell types which have the greatest capacity for LT generation. Interestingly, the LT synthetic capacity of members of one family of myeloid cell, the resident tissue macrophage, varies in a tissuespecific fashion. In particular, pulmonary alveolar macrophages have a far greater LT synthetic capacity than do macrophages from other tissues [8]. In addition, the profile of LTs synthesized, which is dictated by a cell's complement of distal LT synthases, varies with the cell type. Thus, eosinophils and mast cells synthesize predominantly $\mathrm{LTC}_{4}$, while neutrophils synthesize predominantly $\mathrm{LTB}_{4}$. Macrophages synthesize a mixture of $\mathrm{LTC}_{4}$ and $\mathrm{LTB}_{4}$, with differences depending primarily on species, e.g. rat and human macrophages produce predominantly $\mathrm{LTB}_{4}$, while murine macrophages produce predominantly $\mathrm{LTC}_{4}$.

1059 


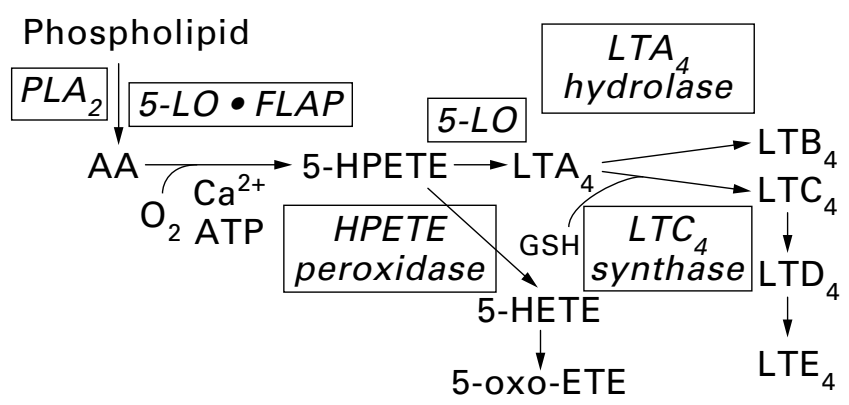

Fig. 1. The 5-LO pathway of arachidonate metabolism. Abbreviations are defined in the text.

Although the LT synthetic capacity of structural or parenchymal cells (epithelial cells, endothelial cells, fibroblasts, smooth muscle cells) is minuscule relative to myeloid cells, they can generate LTs under some circumstances. Importantly, studies with pharmacological inhibitors and antagonists in these cell types suggest that even these low levels of LTs can play important biological roles [9-11]. The distal LT synthase enzymes ( LTA $_{4}$ hydrolase and $\mathrm{LTC}_{4}$ synthase) are expressed somewhat more widely than are 5LO and FLAP, being found in a variety of cell types which lack the latter. Parenchymal cells can also therefore contribute to $\mathrm{LT}$ production by converting $\mathrm{LTA}_{4}$ released by myeloid cells to either $\mathrm{LTB}_{4}$ or $\mathrm{LTC}_{4}$, a process known as 'transcellular' LT synthesis.

\section{Regulation of leukotriene synthesis}

Although LTs must be synthesized de novo, this can be accomplished quite rapidly (i.e. within several minutes) following addition of an agonist, via activation of enzymes which are constitutively present within cells. Activation of both $\mathrm{CPLA}_{2}$ and 5-LO requires an increase in intracellular $\mathrm{Ca}^{2+}$, and the activity of 5-LO is optimized in the presence of ATP. Neither FLAP nor distal LT synthases depends on an 'activation' event, but $\mathrm{LTC}_{4}$ synthase requires reduced glutathione as a cosubstrate. Thus, even the immediate generation of LTs can be influenced by the intracellular levels of the small molecules $\mathrm{Ca}^{2+}$, ATP, and glutathione.

The activities of the LT-synthesizing enzymes can be rapidly augmented by post-translational modifications such as phosphorylation. Phosphorylation of serine 505 on $\mathrm{cPLA}_{2}$, which occurs following the addition of a variety of 'priming' agents, increases the catalytic efficiency with which this enzyme causes hydrolysis of AA [12]. The actions of 5-LO also appear to be augmented by kinase activation [13], and there is some evidence for direct phosphorylation of 5-LO [14].

A delayed type of priming or enhancement of LT synthesis occurs with transcriptional or translational events which increase the steady-state level of key enzyme proteins. This phenomenon has been observed for cPLA $_{2}$ as well as another $\mathrm{Ca}^{2+}$-dependent enzyme, secretory $\mathrm{PLA}_{2}$. It has also been observed for 5-LO and FLAP, and the regulation of expression of these two proteins will be briefly considered here. The promoter region of the 5-LO gene resembles that of 'housekeeping' genes, in that only a few cis-acting elements are present [15]. This is surprising given the restricted cellular and tissue distribution of the encoded protein. Polymorphisms of the 5-LO promoter have recently been described, with mutations resulting in reduced transcription being noted in $\approx 35 \%$ of individuals [16]; it remains to be determined whether these mutations are associated with diminished LT production, or have clinical relevance. In contrast to that for 5-LO, the promoter region of the FLAP gene has multiple regulatory elements [17]. In most experimental systems, FLAP expression is regulated concordantly with 5-LO expression [18], although examples of discordant expression have been described [19]. Despite differences in their promoter structures, expression of both proteins has been reported to be upregulated by similar experimental variables; these include various cytokines, glucocorticoids, and models of macrophage differentiation. It is important to note that the regulatory effects of a given agent can vary depending on the cell type. For example, granulocyte-macrophage colony stimulating factor increases the expression of both 5-LO and FLAP proteins in neutrophils [20], but increases only the expression of $\mathrm{cPLA}_{2}$ in macrophages [21]. Finally, reduced expression of 5-LO and FLAP has been reported in alveolar macrophages obtained from patients infected with the human immunodeficiency virus [22].

In addition to the concentrations of small molecule cofactors, the steady state levels of key proteins, and modifications that alter the catalytic activities of these proteins, one further determinant of LT synthesis has recently been recognized: the intracellular compartmentalization of LT-synthesizing proteins. This has been the focus of extensive investigation in our own and other laboratories in recent years, and a number of surprising findings have been revealed. The remainder of this review will focus on the current state of knowledge in this area.

\section{The translocation model for 5-lipoxygenase activation}

The intracellular locale of the proteins necessary for LT synthesis went largely unstudied for many years. An important advance in our understanding of the mechanism of 5LO activation was the demonstration that the enzyme undergoes a $\mathrm{Ca}^{2+}$-dependent redistribution or translocation from its locale within a soluble intracellular compartment in resting cells to a membrane compartment following agonist 
activation [23]. This process of translocation could reasonably be assumed to bring the enzyme in proximity to its membrane-derived substrate. It was soon determined that cPLA $_{2}$ likewise underwent a $\mathrm{Ca}^{2+}$-dependent redistribution from a soluble to a membrane compartment upon stimulation [24]. Since the helper protein FLAP was also present in the membrane fraction of cells both in the resting and stimulated states [7], a model was formulated in which agonist activation resulted in colocalization of the proteins necessary for arachidonate release and the initiation of LT synthesis. Since LTs were known to be efficiently secreted from cells, the site at which these proteins were colocalized was assumed to be the plasma membrane. However, these early subcellular fractionation studies were not capable of adequately resolving compartmentalization, and there was no a priori reason to do so at the time given the assumptions regarding the primacy of the plasma membrane.

\section{Role of the nuclear envelope in leukotriene synthesis}

\section{(see Fig. 2)}

Surprisingly, when activated blood neutrophils were studied by immunoelectron microscopy, both 5-LO and

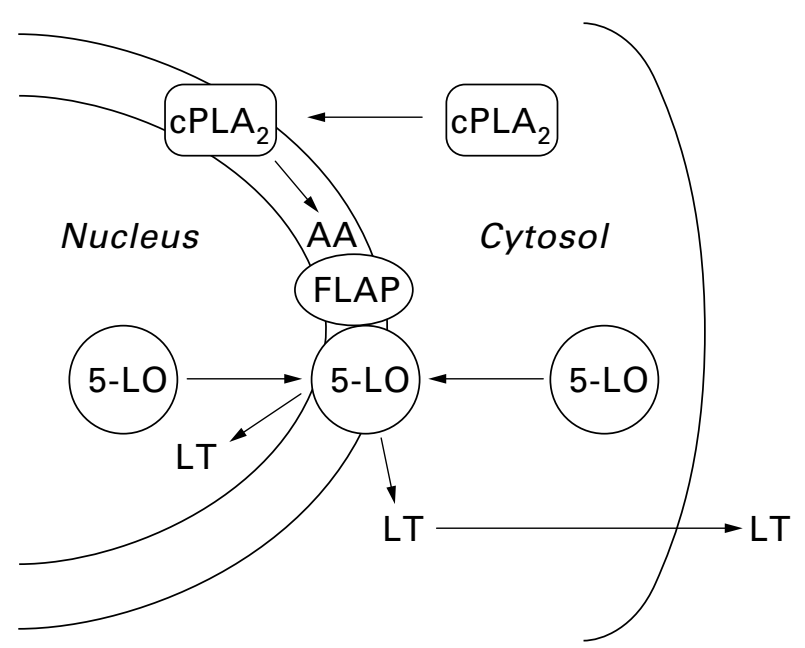

Fig. 2. Role of the nucleus in LT synthesis. LT synthesis requires free AA, hydrolysed from phospholipids by a PLA 2 . Here, arachidonoyl-selective $\mathrm{CPLA}_{2}$ is shown translocating from its resting site in the cytosol to the nuclear envelope upon activation. Arachidonate released from nuclear envelope phospholipids is bound by FLAP, an integral nuclear envelope protein, to facilitate processing by 5-LO. Upon activation, 5-LO translocates from its resting locale(s) in the cytosol and/or nucleus to the nuclear envelope, where it catalyses the initial steps in LT synthesis. Not shown here is LTC $_{4}$ synthase, also an integral nuclear envelope protein. LTs thus synthesized are then capable of either entering the nucleus or being exported out of the cell. Abbreviations are defined in the text.
FLAP were localized to the nuclear envelope [25]. At about the same time, peritoneal macrophages were gently disrupted (so that the plasma membrane but not the nuclear membrane was ruptured) and separated into nuclear, cytosolic, and non-nuclear membrane fractions which were then subjected to immunoblot analysis. FLAP was found predominantly in the nuclear fraction of both resting and stimulated cells; furthermore, 5-LO was found to translocate from the cytosolic to the nuclear fraction upon activation [26]. These results indicating the nuclear envelope to be the site of 5-LO and FLAP colocalization in activated leucocytes appear to reflect a universal phenomenon, now also verified for alveolar macrophages [27,28], blood monocytes [27], mast cells [29], and the rat basophilic leukaemia (RBL) mast cell-like cell line [28]. Localization of 5-LO at the nuclear envelope in activated cells has now also been confirmed in situ, since immunohistochemical analysis revealed an increased number of macrophages with this staining pattern in lung sections from patients with both idiopathic pulmonary fibrosis [3] and primary pulmonary hypertension [4], diseases characterized by constitutive overproduction of LTs.

Of course, these findings with 5-LO and FLAP raised the question of whether $\mathrm{CPLA}_{2}$ translocated to the same membrane. Recent studies using appropriate disruption and fractionation methods as well as immunofluorescence microscopy have indeed revealed that $\mathrm{CPLA}_{2}$ is also localized primarily at the nuclear envelope in a variety of types of stimulated cells [26,30,31]. Importantly, translocation of cPLA $_{2}$ to the nuclear envelope has been shown to be associated with selective hydrolysis of nuclear membrane phospholipids [30]. Recently, LTC $_{4}$ synthase has been shown to have a high degree of homology with FLAP; like FLAP, it too is an integral membrane protein located primarily at the nuclear envelope [32]. Taken together, there is now abundant evidence suggesting that the nuclear envelope is the site at which AA release (at least that mediated by $\mathrm{CPLA}_{2}$ ), $\mathrm{LTA}_{4}$ synthesis, and $\mathrm{LTA}_{4}$ conversion to $\mathrm{LTC}_{4}$ all occur. The mechanism by which the translocation of $\mathrm{CPLA}_{2}$ and 5-LO which originate in the cytosol is targeted to the nuclear envelope, as opposed to other intracellular membranes, remains to be elucidated. In any case, these findings raise the important question of why mediators destined for extracellular secretion would be synthesized deep within the cell, and this will be considered at the end of this communication.

\section{Cell-specific compartmentalization of 5-lipoxygenase in resting cells}

\section{(see Fig. 2)}

Initial studies in unstimulated blood neutrophils [33] and peritoneal macrophages [26] demonstrated that 5-LO was 
predominantly cytosolic, and this finding has been extended to blood monocytes [27]. Unexpectedly, subsequent fractionation as well as immunomicroscopic studies of isolated alveolar macrophages [27,28], mast cell-like RBL cells [33], and primary mast cells [29] revealed abundant intranuclear 5-LO in addition to that found within the cytosol. Importantly, an intranuclear pool of 5-LO in alveolar macrophages has been confirmed in situ by immunohistochemical staining of normal human lung tissue [3]. A portion of the intranuclear pool in RBL cells is insoluble and has biochemical characteristics suggesting that it is chromatin-associated [33]. Immunoelectron microscopic analysis of human alveolar macrophages demonstrated that intranuclear 5-LO was not randomly distributed, but was instead concentrated in the euchromatin region [27], that portion of the nucleus where actively transcribing genes are distributed. That the intranuclear pool of 5-LO participates in cellular LT synthesis is suggested by the facts that it is catalytically active in cell-free assays and translocates to the nuclear envelope upon agonist stimulation [28]. These findings indicate that compartmentalization of 5-LO in unstimulated cells varies depending on the cell type, with some cells exhibiting exclusively cytosolic enzyme and others containing both cytosolic and intranuclear pools; in either case, activation is associated with translocation to the nuclear envelope. The mechanisms by which compartmentalization of 5-LO is differentially regulated in different cell types are not currently understood.

\section{Dynamic regulation of 5-lipoxygenase compartmentalization}

Rather than a static model in which 5-LO compartmentalization is considered to be dictated solely by cell type, several lines of evidence indicate that it is, in fact, a dynamically regulated process even within a given cell type. The first is based on data obtained with various mononuclear phagocyte populations. Blood monocytes, the precursors for all mature tissue macrophages, contain cytosolic 5-LO [27]. While mature peritoneal macrophages retain this cytosolic distribution [26], alveolar macrophages do not [27,28], indicating that nuclear import of this protein accompanies monocyte migration into the pulmonary alveolar space, but not the peritoneum. Second, the recruitment of blood neutrophils into sites of inflammation (either pulmonary alveolus or peritoneum) is associated with rapid movement of 5-LO into the nucleus, which is not itself accompanied by LT synthesis [34]. This can be mimicked by adherence of blood neutrophils to various surfaces [34]. A final example involves DMSO-induced differentiation of the promyelocytic leukaemic cell line, HL-60, into granulocytic cells, a process previously recognized [35] to be associated with increased expression of both 5-LO and FLAP. We have observed that incubation of these cells with serum results in a shift of 5-LO localization from the cytosol to the intranuclear compartment (unpublished results). Again, the molecular mechanisms by which dynamic nuclear import of 5-LO is mediated are as yet undefined. However, it is interesting that experimental conditions in which nuclear import of both $\mathrm{cPLA}_{2}$ [36] and cyclo-oxygenase-2 [37], an inducible form of the enzyme responsible for conversion of AA to prostaglandins, have recently been described. Thus, the phenomenon of dynamic modulation of 5-LO compartmentalization may serve as a model which also applies to other enzymes in the eicosanoid synthetic pathway.

\section{Metabolic implications of 5-lipoxygenase localization}

The fact that 5-LO and FLAP (along with other relevant proteins) are localized to the nuclear envelope of activated cells implies that LT synthesis is initiated at this site. As a result, the local concentrations of LTs within the nucleus are likely to be quite high. Teleologically, these observations strongly suggest that the potential nuclear actions of these bioactive lipid mediators may be more important than those extracellular actions which have been classically recognized. Indeed, a growing body of evidence implicates endogenously produced 5-LO metabolites as modulators of such fundamental processes as mitogenesis [11], apoptosis [2,38], and the expression or activation of various transcription factors including $\mathrm{N} F_{\kappa} \mathrm{B}[10,39,40]$ and fos [11]. Theoretically, these actions of LTs could involve their direct interaction with nucleic acids, transcription factors, or signalling pathways. The recent identification of a nuclear receptor for $\mathrm{LTB}_{4}$ exemplifies such a direct intranuclear interaction [41]; interestingly, this receptor was a member of the steroid superfamily of transcription factors and its ligation was capable of inducing gene transcription. Finally, it should also be recalled that reactive oxygen species are a byproduct of arachidonate 5-lipoxygenation, and these reactive intermediates could themselves possess nuclear actions by activating transcription factors or otherwise modifying nuclear constituents. 5-LO-dependent activation of $\mathrm{N} F_{\kappa} \mathrm{B}$ has been ascribed to reactive oxygen species in some [39,40] but not all [10] experimental systems.

The propensity of particular LT-forming proteins to be localized at particular intracellular sites raises the possibility that metabolic coupling among proteins will be dictated by their topographic proximity. This notion posits that both the access of 5-LO/FLAP to free AA and their capacity to supply $\mathrm{LTA}_{4}$ for LT synthesis will be enhanced by the proximity of $\mathrm{PLA}_{2}$ and LT synthases, respectively, to the nuclear envelope. In this regard, it has long been recognized that different functional pools of AA exist within a given cell type, and different metabolic fates for AA could reflect 
the topographic proximity of $\mathrm{PLA}_{2} \mathrm{~S}$ and downstream enzymes.

Dynamic nuclear import of 5-LO adds a further measure of complexity to the regulation of LT synthesis. It should be emphasized that nuclear import is not itself associated with LT synthesis. But how does this phenomenon in resting cells influence subsequent LT generation upon activation? In two different experimental models (peritoneal vs alveolar macrophages and blood vs. recruited neutrophils), the latter cells with intranuclear 5-LO require a higher dose of ionophore to trigger LT synthesis than do the former cells with cytosolic enzyme $[8,34]$. This could reflect the likelihood that intranuclear $\mathrm{Ca}^{2+}$ concentrations are lower than cytosolic $\mathrm{Ca}^{2+}$ concentrations following the addition of an extracellular stimulus. However, alveolar macrophages [8], recruited neutrophils [34], and serum-treated differentiated HL-60 cells (unpublished results) (all with intranuclear 5LO) all display greater maximal capacities for LT production in response to stimuli than do the corresponding cells with cytosolic 5-LO. The lower sensitivity and greater capacity of these cells with an intranuclear distribution of 5-LO could alternatively reflect a lower hydroperoxide tone in the nuclear than cytosolic compartment. In any case, a growing body of evidence indicates that compartmentalization of 5-LO is an important determinant of LT synthetic responses.

\section{Non-metabolic implications of 5-lipoxygenase localization}

The localization of 5-LO could have biological implications which go beyond its catalytic products, LTs and reactive oxygen species. The 5-LO protein has a Src homology-3 binding motif [42], which could mediate protein-protein interactions between the enzyme and Src homology-3 domains. These domains are typically present in proteins which are substrates for tyrosine phosphorylation, and purified 5-LO has been shown to bind with certain cytoskeletal proteins [42]. This motif on 5-LO has been suggested to mediate enzyme translocation in response to activation [14]. The facts that 5-LO can be found within the euchromatin region of nuclei and can be demonstrated to be chromatin-associated further suggest that the enzyme could likewise interact directly with intranuclear proteins or, perhaps, genes.

\section{Conclusions}

Our understanding of the molecular mechanisms and regulation of LT synthesis has increased dramatically in recent years. In particular, investigations into the cell biology of this metabolic pathway have revealed an unexpected role for the nucleus. While many questions of a molecular and functional nature remain to be answered, this finding provides the impetus to explore novel intracellular actions of LTs beyond those traditionally appreciated.

\section{References}

1 Lewis RA, Austen KF, Soberman RJ. Leukotrienes and other products of the 5-lipoxygenase pathway: biochemistry and relation to pathobiology in human disease. $\mathrm{N}$ Engl $\mathrm{J}$ Med 1990; 323:645-55.

2 Avis I, Jett M, Boyle T, Vos M, Moody T, Treston A, Martinez A, Mulshine J. Growth control of lung cancer by interruption of 5-lipoxygenase-mediated growth factor signaling. J Clin Invest 1996; 97:806-13.

3 Wilborn J, Bailie M, Coffey M, Burdick M, Strieter R, PetersGolden M. Constitutive activation of 5-lipoxygenase in the lungs of patients with idiopathic pulmonary fibrosis. J Clin Invest 1996; 97:1827-36.

4 Wright L, Tuder R, Wang J, Cool C, Lepley R, Voelkel N. 5Lipoxygenase and 5-lipoxygenase activating protein (FLAP) immunoreactivity in lungs from patients with primary pulmonary hypertension. Am J Respir Crit Care Med 1998; 157:219-29.

5 Bailie M, Standiford T, Laichalk L, Coffey M, Strieter R, Peters-Golden M. Leukotriene-deficient mice manifest enhanced lethality from Klebsiella pneumonia in association with decreased alveolar macrophage phagocytic and bactericidal activities. J Immunol 1996; 157:5221-4.

6 Dennis E. Diversity of group types, regulation, and function of phospholipase A $_{2}$. J Biol Chem 1994; 269:13057-60.

7 Miller DK, Gillard JW, Vickers PJ, Sadowski S, Leveille C, Mancini JA, Charleson P, Dixon RAF, Ford-Hutchinson AW, Fortin R, Gauthier JY, Rodkey J, Rosen R, Rouzer C, Sigal IS, Strader CD, Evans JF. Identification and isolation of a membrane protein necessary for leukotriene production. Nature 1990; 343:278-81.

8 Peters-Golden M, McNish RW, Hyzy R, Shelly C, Toews GB. Alterations in the pattern of arachidonate metabolism accompany rat macrophage differentiation in the lung. J Immunol 1990; 144:263-70.

9 Peppelenbosch M, Tertoolen L, den Hertog J, de Laat S. Epidermal growth factor activates calcium channels by phospholipase $\mathrm{A}_{2} / 5$-lipoxygenase-mediated leukotriene $\mathrm{C}_{4}$ production. Cell 1992; 69:295-303.

10 Lee S, Felts K, Parry G, Armacost L, Cobb R. Inhibition of 5 -lipoxygenase blocks IL- $1 \beta$-induced vascular adhesion molecule-1 gene expression in human endothelial cells. J Immunol 1997; 158:3401-7.

11 Beno D, Mullen J, Davis B. Lipoxygenase inhibitors block PDGF-induced mitogenesis: a MAPK-independent mechanism that blocks fos and egr. Am J Physiol 1995; 268:C604-10.

12 Kramer R, Roberts E, Manetta J, Hyslop P, Jakubowski J. Thrombin-induced phosphorylation and activation of $\mathrm{Ca}^{2+}$ sensitive cytosolic phospholipase $\mathrm{A}_{2}$ in human platelets. J Biol Chem 1993; 268:26795-804.

13 Peters-Golden M, McNish R, Sporn P, Balazovich K. Basal activation of protein kinase $\mathrm{C}$ in rat alveolar macrophages: 
implications for arachidonate metabolism. Am J Physiol: Lung Cell Mol Physiol 1991; 261:L462-L471.

14 Lepley R, Muskardin D, Fitzpatrick F. Tyrosine kinase activity modulates catalysis and translocation of cellular 5lipoxygenase. J Biol Chem 1996; 271:6179-84.

15 Hoshiko S, Radmark O, Samuelsson B. Characterization of the human 5-lipoxygenase gene promoter. Proc Natl Acad Sci USA 1990; 87:9073-7.

16 In K, Asano K, Beier D, Grobholz J, Finn P, Silverman E, Silverman E, Collins T, Fischer A, Keith T, Serino K, Kim S, De Sanctis G, Yandava C, Pillari A, Rubin P, Kemp J, Israel E, Busse W, Ledford D, Murray J, Segal A, Tinkleman D, Drazen J. Naturally occurring mutations in the human 5lipoxygenase gene promoter that modify transcription factor binding and reporter gene transcription. J Clin Invest 1997; 99:1130-7.

17 Kennedy B, Diehl R, Boie Y, Adam M, Dixon R. Gene characterization and promoter analysis of the human 5lipoxygenase-activating protein (FLAP). J Biol Chem 1991; 266:8511-6.

18 Reid GK, Kargman S, Vickers PJ, Mancini JA, Leveille C, Ethier D, Miller DK, Gillard JW, Dixon RAF, Evans JF. Correlation between expression of 5-lipoxygenase-activating protein, 5-lipoxygenase, and cellular leukotriene synthesis. J Biol Chem 1990; 265:19818-23.

19 Jakobsson P, Steinhilber D, Odlander B, Radmark O, Claesson $\mathrm{H}$, Samuelsson B. On the expression and regulation of 5lipoxygenase in human lymphocytes. Proc Natl Acad Sci USA 1992; 89:3521-5.

20 Pouliot M, McDonald P, Borgeat P, McColl S. Granulocyte/ macrophage colony-stimulating factor stimulates the expression of the 5-lipoxygenase-activating protein (FLAP) in human neutrophils. J Exp Med 1994; 179:1225-32.

21 Brock TG, McNish RW, Coffey MJ, Ojo TC, Phare SM, Peters-Golden M. Effect of granulocyte-macrophage colonystimulating factor on eicosanoid production by mononuclear phagocytes. J Immunol 1996; 156:2522-7.

22 Coffey M, Phare S, Kazanjian P, Peters-Golden M. 5Lipoxygenase metabolism in alveolar macrophages from subjects infected with the human immunodeficiency virus. J Immunol 1996; 157:393-9.

23 Rouzer CA, Kargman S. Translocation of 5-lipoxygenase to the membrane in human leukocytes challenged with ionophore A23187. J Biol Chem 1988; 263:10980-8.

24 Channon J, Leslie C. A calcium-dependent mechanism for associating a soluble arachidonoyl-hydrolyzing phospholipase A2 with membrane in the macrophage cell line RAW 264. 7 J Biol Chem 1990; 265:5409-13.

25 Woods J, Evans J, Ethier D, Scott S, Vickers P, Hearn L, Charleson S, Heibein J, Singer I. 5-Lipoxygenase and 5lipoxygenase activating protein are localized in the nuclear envelope of activated human leukocytes. J Exp Med 1993; 178:1935-46.

26 Peters-Golden M, McNish R. Redistribution of 5-lipoxygenase and cytosolic phospholipase $\mathrm{A}_{2}$ to the nuclear fraction upon macrophage activation. Biochem Biophys Res Commun 1993; 196:147-53.
27 Woods J, Coffey M, Brock T, Singer I, Peters-Golden M. 5Lipoxygenase is located in the euchromatin of the nucleus in resting human alveolar macrophages and translocates to the nuclear envelope upon cell activation. J Clin Invest 1995; 95:2035-40.

28 Brock TG, McNish RW, Peters-Golden M. Translocation and leukotriene synthetic capacity of nuclear 5-lipoxygenase in rat basophilic leukemia cells and alveolar macrophages. J Biol Chem 1995; 270:21652-8.

29 Chen X-S, Naumann T, Kurre U, Jenkins N, Copeland N, Funk C. cDNA cloning, expression, mutagenesis, intracellular localization, and gene chromosomal assignment of mouse 5lipoxygenase. J Biol Chem 1995; 270:17993-9.

30 Peters-Golden M, Song K, Marshall T, Brock T. Translocation of cytosolic phospholipase $\mathrm{A}_{2}$ to the nuclear envelope elicits topographically localized phospholipid hydrolysis. Biochem J 1996; 318:797-803.

31 Glover S. Bayburt T, Jonas M, Chi E, Gelb M. Translocation of the $85-\mathrm{kDa}$ phospholipase A2 from cytosol to the nuclear envelope in rat basophilic leukemia cells stimulated with calcium ionophore or IgE/antigen. J Biol Chem 1995; 270: 15359-67.

32 Penrose J, Spector J, Lam B, Friend D, Xu K, Jack R, Austen K. Purification of human lung $\mathrm{LTC}_{4}$ synthase and preparation of a polyclonal antibody. Am J Respir Crit Care Med 1995; 152:283-9.

33 Brock TG, Paine R, Peters-Golden M. Localization of 5lipoxygenase to the nucleus of unstimulated rat basophilic leukemia cells. J Biol Chem 1994; 269:22059-66.

34 Brock T, McNish R, Bailie M, Peters-Golden M. Rapid import of cytosolic 5-lipoxygenase into the nucleus of neutrophils after in vivo recruitment and in vitro adherence. J Biol Chem 1997; 272:8276-80.

35 Kargman S, Rouzer CA. Studies on the regulation, biosynthesis, and activation of 5-lipoxygenase in differentiated HL60 cells. J Biol Chem 1989; 264:13313-20.

36 Sierra-Honigmann M, Bradley J, Pober J. 'Cytosolic' phospholipase A2 is in the nucleus of subconfluent endothelial cells but confined to the cytoplasm of confluent endothelial cells and redistributes to the nuclear envelope and cell junctions upon histamine stimulation. Lab Invest 1996; 74:684-95.

37 Coffey R, Hawkey C, Damstrup L, Graves-Deal R, Daniel V, Dempsey P, Chinery R, Kirkland S, DuBois R, Jetton T, Morrow J. Epidermal growth factor receptor activation induces nuclear targeting of cyclooxygenase-2, basolateral release of prostaglandins, and mitogenesis in polarizing colon cancer cells. Proc Natl Acad Sci USA 1997; 94:657-62.

38 Hebert M-J, Takano T, Holthofer H, Brady H. Sequential morphologic events during apoptosis of human neutrophils. Modulation by lipoxygenase-derived eicosanoids. J Immunol 1996; 157:3105-15.

39 Bonizzi G, Piette J, Merville M-P, Bours V. Distinct signal transduction pathways mediate nuclear factor- $\kappa \mathrm{B}$ induction by IL-1 $\beta$ in epithelial and lymphoid cells. J Immunol 1997; 159:5264-72.

40 Los M, Schenk H, Hexel K, Baeuerle P, Droge W, SchulzeOsthoff K. IL-2 gene expression and $\mathrm{NF}^{-} \mathrm{B}$ activation through 
CD28 requires reactive oxygen production by 5-lipoxygenase. EMBO J 1995; 14:3731-40.

41 Devchand P, Keller H, Peters J, Vazquez M, Gonzalez F, Wahli $\mathrm{W}$. The PPAR $\alpha$-leukotriene $\mathrm{B}_{4}$ pathway to inflammation control. Nature 1996; 384:39-43.
42 Lepley RA, Fitzpatrick F. 5-lipoxygenase contains a functional Src homology 3-binding motif that interacts with the Src homology 3 domain of Grb2 and cytoskeletal proteins. J Biol Chem 1994; 269:24163-8. 